\title{
Global attractivity of a nonautonomous discrete logistic model
}

\author{
Qinqin ZHANG and Zhan ZHOU
}

(Received October 14, 1998; Revised February 10, 1999)

Abstract. In this paper we consider the nonautonomous discrete logistic model

$$
x_{n+1}=x_{n} \exp \left[r_{n}\left(1-x_{n}\right)\right], \quad n \in N
$$

where $\left\{r_{n}\right\}$ is a sequence of nonnegative numbers. We obtain some sufficient conditions for an arbitrary solution $\left\{x_{n}\right\}$ satisfying the initial condition

$$
x_{0}=a>0
$$

to converge to 1 as $n \rightarrow \infty$. Under appropriate hypotheses, the necessary and sufficient conditions for any solution of (1.1) with (1.2) tending to 1 as $n \rightarrow \infty$ have also been obtained.

Key words: discrete nonautonomous logistic model, global attractivity.

\section{Introduction}

Consider the discrete nonautonomous logistic model

$$
x_{n+1}=x_{n} \exp \left[r_{n}\left(1-x_{n}\right)\right], \quad n \in N,
$$

where $\left\{r_{n}\right\}$ is a sequence of nonnegative numbers. It is easy to see that, for any given initial condition

$$
x_{0}=a>0,
$$

Eq. (1.1) has an unique solution $\left\{x_{n}\right\}$ which is positive for all $n \in N$ and satisfies (1.2). In [1], it was proved that every solution of (1.1) with (1.2) tends to 1 if $r_{n} \leq 3 / 2$ and $\sum_{n=0}^{\infty} r_{n}=\infty$.

When $r_{n} \equiv r>0$, Eq. (1.1) reduces to

$$
x_{n+1}=x_{n} \exp \left[r\left(1-x_{n}\right)\right], \quad n \in N,
$$

1991 Mathematics Subject Classification : 39A10, 39A12.

This work was partially supported by NNSF of China. 
which has been studied in the literature in its own right as a discrete population model of a single species with non-overlapping generations. It was shown in $[2,3]$ that for some values of the parameter $r$, solutions of Eq. (1.3) are "chaotic". It was also proved in [4] that any solution of Eq. (1.3) with (1.2) converges to 1 as $n \rightarrow \infty$ if and only if $r \leq 2$.

In this paper, we discuss Eq. (1.1) and obtain the following results.

\section{Theorem 1.1 If}

$$
\sum_{n=0}^{\infty} r_{n}=\infty
$$

and

$$
\limsup _{n \rightarrow \infty} r_{n} \leq 2 \text {. }
$$

Then any solution $\left\{x_{n}\right\}$ of Eq. (1.1) with (1.2) converges to 1 as $n \rightarrow \infty$.

Theorem 1.2 Assume that $\left\{r_{n}\right\}$ is bounded. If

$$
\liminf _{n \rightarrow \infty} r_{n}>2
$$

then every nontrivial solution $\left\{x_{n}\right\}$ of $E q$. (1.1) with (1.2) cannot converge to 1 as $n \rightarrow \infty$.

Combining Theorem 1.1 and 1.2, we obtain the following necessary and sufficient conditions, that is

Corollary 1.1 Assume that (1.4) holds and the limit $\lim _{n \rightarrow \infty} r_{n}$ exists. Then any solution $\left\{x_{n}\right\}$ of Eq. (1.1) with (1.2) converges to 1 as $n \rightarrow \infty$ if and only if

$$
\lim _{n \rightarrow \infty} r_{n} \leq 2 .
$$

\section{Proofs of Theorem 1.1 and $\mathbf{1 . 2}$}

First, we establish the following lemma.

Lemma 2.1 Assume that $r$ is a nonnegative constant, let

$$
f(x)=x(\exp [r(1-x)]+1)-2 .
$$


If there exists a constant $x^{*}$ such that

$$
f\left(x^{*}\right)\left(x^{*}-1\right)<0
$$

then

$$
\left(x^{*}-1\right)^{2}<\frac{3}{2}(r-2) \text {. }
$$

Proof. Clearly, $f(1)=0, f(x) \leq-2$ for $x \leq 0$, and $f(x) \geq 0$ for $x \geq 2$. Since (2.1) holds, we see that either $x^{*} \in(0,1)$ or $x^{*} \in(1,2)$.

If $x^{*} \in(0,1)$, by $(2.1)$, we know that $f\left(x^{*}\right)>0$, this implies

$$
\begin{aligned}
r & >\frac{1}{1-x^{*}} \ln \frac{2-x^{*}}{x^{*}} \\
& =\frac{1}{1-x^{*}} \ln \frac{1+\left(1-x^{*}\right)}{1-\left(1-x^{*}\right)} \\
& =\frac{1}{1-x^{*}}\left(\sum_{k=1}^{\infty}(-1)^{k+1} \frac{\left(1-x^{*}\right)^{k}}{k}+\sum_{k=1}^{\infty} \frac{\left(1-x^{*}\right)^{k}}{k}\right) \\
& \geq \frac{2}{1-x^{*}}\left(\left(1-x^{*}\right)+\frac{\left(1-x^{*}\right)^{3}}{3}\right) \\
& =2+\frac{2}{3}\left(1-x^{*}\right)^{2},
\end{aligned}
$$

which leads to (2.2).

If $x^{*} \in(1,2)$, by $(2.1)$, we know that $f\left(x^{*}\right)<0$, this implies that

$$
\begin{aligned}
r & >\frac{1}{x^{*}-1} \ln \frac{x^{*}}{2-x^{*}} \\
& =\frac{1}{x^{*}-1} \ln \frac{1+\left(x^{*}-1\right)}{1-\left(x^{*}-1\right)} \\
& \geq 2+\frac{2}{3}\left(x^{*}-1\right)^{2} .
\end{aligned}
$$

So, $(2.2)$ holds.

The proof of Lemma 1.1 is now complete.

Proof of Theorem 1.1. Assume that $\left\{x_{n}\right\}$ is a solution of Eq. (1.1) with (1.2). Let

$$
V(n)=\left(x_{n}-1\right)^{2}, \quad n \in N .
$$


Then

$$
\begin{aligned}
\triangle V(n) & =\left(x_{n+1}-1\right)^{2}-\left(x_{n}-1\right)^{2} \\
& =\left(x_{n+1}-x_{n}\right)\left(x_{n+1}+x_{n}-2\right) \\
& =x_{n}\left(\exp \left[r_{n}\left(1-x_{n}\right)\right]-1\right)\left(x_{n}\left(\exp \left[r_{n}\left(1-x_{n}\right)\right]+1\right)-2\right)
\end{aligned}
$$

here $\triangle$ denotes the forward difference operator defined by $\triangle V(n)=V(n+$ 1) $-V(n)$.

Since

$$
\left(\exp \left[r_{n}\left(1-x_{n}\right)\right]-1\right)\left(x_{n}-1\right) \leq 0, \quad n \in N .
$$

We claim that, for any $m \in N$,

$$
\triangle V(m)>0 \text { implies } V(m)<\frac{3}{2}\left(r_{m}-2\right) .
$$

In fact, if $\triangle V(m)>0$, by (2.4) and (2.5), we have

$$
\left(x_{m}\left(\exp \left[r_{m}\left(1-x_{m}\right)\right]+1\right)-2\right)\left(x_{m}-1\right)<0,
$$

this leads to, by Lemma 1.1, that

$$
\left(x_{m}-1\right)^{2}<\frac{3}{2}\left(r_{m}-2\right) .
$$

So (2.6) holds.

We consider three possible cases.

Case 1: There is a $n^{*} \in N$, such that $\triangle V(n)>0$ for $n \geq n^{*}$.

In this case, by (2.6), we have

$$
V(n)<\frac{3}{2}\left(r_{n}-2\right) \text { for } n \geq n^{*}
$$

By this and (1.5), we know that $\lim _{\sup _{n \rightarrow \infty}} V(n) \leq 0$. Since $V(n) \geq 0$ for $n \in N$, we see that $\lim _{n \rightarrow \infty} V(n)=0$, which is equivalent to $\lim _{n \rightarrow \infty} x_{n}=1$.

Case 2: There is a $n^{*} \in N$, such that $\triangle V(n) \leq 0$ for $n \geq n^{*}$.

In this case, $\{V(n)\}$ is nonincreasing for $n \geq n^{*}$. Since $V(n) \geq 0$, we see that $\lim _{n \rightarrow \infty} V(n)$ exists. Let

$$
\alpha=\lim _{n \rightarrow \infty} V(n),
$$


then

$$
\lim _{n \rightarrow \infty}\left|x_{n}-1\right|=\sqrt{\alpha}
$$

Denote $\beta=\sqrt{\alpha}$, we shall prove that $\beta=0$.

In fact, if $\beta>0$, we consider three subcases.

Subcase a: $x_{n}-1>0$ for large $n$.

In this subcase, by (2.8), we have

$$
\lim _{n \rightarrow \infty} x_{n}=1+\beta \text {. }
$$

There is a sufficient large integer $m_{1}$, such that

$$
x_{n}-1 \geq \frac{\beta}{2} \quad \text { for } \quad n \geq m_{1} \text {. }
$$

By (1.1), we get

$$
x_{n+1}=x_{n} \exp \left[r_{n}\left(1-x_{n}\right)\right] \leq x_{n} \exp \left[-\frac{\beta}{2} r_{n}\right] \text { for } n \geq m_{1},
$$

this leads to, for $p \in N$, that

$$
x_{m_{1}+p+1} \leq x_{m_{1}} \exp \left[-\frac{\beta}{2} \sum_{i=m_{1}}^{m_{1}+p} r_{i}\right] \text {. }
$$

Let $p \rightarrow \infty$ in (2.11), and noting (1.4), we get

$$
\lim _{p \rightarrow \infty} x_{m_{1}+p+1}=0 .
$$

This contradicts (2.9), so subcase a is impossible.

Subcase b: $x_{n}-1<0$ for large $n$.

In this case, by (2.8), we have

$$
\lim _{n \rightarrow \infty} x_{n}=1-\beta \text {. }
$$

There is a sufficient large integer $m_{2}$ such that

$$
1-x_{n} \geq \frac{\beta}{2} \text { for } n \geq m_{2}
$$

By (1.1), we get

$$
x_{n+1}=x_{n} \exp \left[r_{n}\left(1-x_{n}\right)\right] \geq x_{n} \exp \left[\frac{\beta}{2} r_{n}\right] \text { for } n \geq m_{2} .
$$


So, for $p \in N$, that

$$
x_{m_{2}+p+1} \geq x_{m_{2}} \exp \left[\frac{\beta}{2} \sum_{i=m_{2}}^{m_{2}+p} r_{i}\right] \text {. }
$$

Let $p \rightarrow \infty$ in (2.14), and noting (1.4), we are led to

$$
\lim _{p \rightarrow \infty} x_{m_{2}+p+1}=\infty
$$

which contradicts (2.12). So subcase b is impossible.

Subcase c: There is a sequence $\left\{n_{i}\right\}$ of positive integers, such that

$$
x_{n_{i}}-1<0, \quad x_{n_{i}+1}-1>0 .
$$

Thus

$$
\lim _{i \rightarrow \infty} x_{n_{i}}=1-\beta, \quad \lim _{i \rightarrow \infty} x_{n_{i}+1}=1+\beta
$$

Since

$$
x_{n_{i}+1}=x_{n_{i}} \exp \left[r_{n_{i}}\left(1-x_{n_{i}}\right)\right],
$$

by $(2.15)$, we have

$$
\begin{aligned}
\lim _{i \rightarrow \infty} r_{n_{i}} & =\lim _{i \rightarrow \infty} \frac{1}{1-x_{n_{i}}} \ln \frac{x_{n_{i}+1}}{x_{n_{i}}} \\
& =\frac{1}{\beta} \ln \frac{1+\beta}{1-\beta} \\
& =\frac{1}{\beta}\left(\sum_{k=1}^{\infty}(-1)^{k+1} \frac{\beta^{k}}{k}+\sum_{k=1}^{\infty} \frac{\beta^{k}}{k}\right) \\
& =2 \sum_{k=0}^{\infty} \frac{\beta^{2 k}}{2 k+1} \\
& >2 .
\end{aligned}
$$

This contradicts (1.5), and subcase $\mathrm{c}$ is impossible.

According to the above discussions, we know that $\beta=0$ and so $\lim _{n \rightarrow \infty} x_{n}=1$ for case 2 .

Case 3: There exists a sequence $\left\{n_{j}\right\}$ of integers, such that

$$
\triangle V\left(n_{1}\right) \leq 0, \quad \triangle V(n)>0 \text { for } n_{2 k-1}+1 \leq n \leq n_{2 k},
$$




$$
\triangle V(n) \leq 0 \text { for } n_{2 k}+1 \leq n \leq n_{2 k+1}, \quad k=1,2, \ldots .
$$

In this case, we see that

$$
V(n) \leq V\left(n_{2 k}+1\right) \text { for } n_{2 k-1}+1 \leq n \leq n_{2 k+1}, \quad k=1,2, \ldots .
$$

Since $\Delta V\left(n_{2 k}\right)>0$ for $k=1,2, \ldots$, by $(2.6)$, we get

$$
V\left(n_{2 k}\right)<\frac{3}{2}\left(r_{n_{2 k}}-2\right), \quad k=1,2, \ldots .
$$

By (1.5), this implies that $\lim _{k \rightarrow \infty} V\left(n_{2 k}\right)=0$, that is,

$$
\lim _{k \rightarrow \infty} x_{n_{2 k}}=1 .
$$

By (1.1),

$$
x_{n_{2 k}+1}=x_{n_{2 k}} \exp \left[r_{n_{2 k}}\left(1-x_{n_{2 k}}\right)\right],
$$

so

$$
\lim _{k \rightarrow \infty} x_{n_{2 k}+1}=1 \text { and } \lim _{k \rightarrow \infty} V\left(n_{2 k}+1\right)=0 .
$$

Noting (2.16), it is obvious that

$$
\lim _{n \rightarrow \infty} V(n)=0, \text { i.e. } \lim _{n \rightarrow \infty} x_{n}=1 .
$$

In view of the above three cases, we know that Theorem 1.1 holds. This completes the proof.

Proof of Theorem 1.2. Assume, for the sake of contradiction, that (1.1) has a nontrivial solution $\left\{x_{n}\right\}$ such that $\lim _{n \rightarrow \infty} x_{n}=1$.

By (1.1), we have

$$
x_{n+1}-1=\left(x_{n}-1\right) \exp \left[r_{n}\left(1-x_{n}\right)\right]+\exp \left[r_{n}\left(1-x_{n}\right)\right]-1,
$$

so,

$$
\frac{\left|x_{n+1}-1\right|}{\left|x_{n}-1\right|} \geq \frac{\left|\exp \left[r_{n}\left(1-x_{n}\right)\right]-1\right|}{\left|r_{n}\left(1-x_{n}\right)\right|} r_{n}-\exp \left[r_{n}\left(1-x_{n}\right)\right] .
$$

Since $\left\{r_{n}\right\}$ is bounded, we know $\lim _{n \rightarrow \infty} r_{n}\left(1-x_{n}\right)=0$. By $(2.19)$ and (1.6), we get

$$
\liminf _{n \rightarrow \infty} \frac{\left|x_{n+1}-1\right|}{\left|x_{n}-1\right|} \geq \liminf _{n \rightarrow \infty} r_{n}-1>1,
$$


which leads to

$$
\lim _{n \rightarrow \infty}\left|x_{n}-1\right|=\infty
$$

This is a contradiction. Therefore, Theorem 1.2 holds, the proof is complete.

Acknowledgments We would like to thank the referee for his helpful suggestions.

\section{References}

[1] So J.W.-H. and Yu J.S., Global stability in a logistic equation with piecewise constant arguments. Hokkaido Math. J. 24 (1995), 269-286.

[2] May R.M., Biological populations obeying difference equations: stable points, stable cycles and chaos. J. Theo. Biol. 51 (1975), 511-524.

[3] May R.M. and Oster G.F., Bifurcations and dynamics complexity in simple ecological models. Amer. Nut. 110 (1976), 573-599.

[4] Agarwal R.P., Difference Equations and Inequalities: Theory, Method and Applications. Marcel Dekker, New York, 1992.

Qinqin Zhang

Department of Applied Mathematics Hunan University

Changsha, Hunan 410082

P. R. China

Zhan Zhou

Department of Applied Mathematics

Hunan University

Changsha, Hunan 410082

P. R. China

E-mail: zzhou@mail.hunu.edu.cn 